SSFB/CPP-05-96

TTP05-24

\title{
Recent progress in computing four-loop massive correlators *
}

\author{
K. G. Chetyrkin ${ }^{\mathrm{a}}$, J. H. Kühn ${ }^{\mathrm{a}}$ and C. Sturm ${ }^{\mathrm{ab}}$ \\ a Institut für Theoretische Teilchenphysik, Universität Karlsruhe, D-76128 Karlsruhe, Germany \\ ${ }^{\text {b}}$ Dipartimento di Fisica Teorica, Università di Torino, I-10125 Torino, Italy \& INFN, Sezione di Torino
}

\begin{abstract}
We report about recent progress in computing four-loop massive correlators. The expansion of these correlators in the external momentum leads to vacuum integrals. The calculation of these vacuum integrals can be used to determine Taylor expansion coefficients of the vacuum polarization function and decoupling functions in perturbative Quantum chromodynamics. New results at four-loop order for the lowest Taylor expansion coefficient of the vacuum polarization function and for the decoupling relation are presented.
\end{abstract}

\section{Introduction}

Two-point correlators have been studied in great detail in the framework of perturbative quantum field theory. Due to simple kinematics (only one external momentum) even multi-loop calculations can be performed. The results for all physically interesting diagonal and non-diagonal correlators and including full quark mass dependence are available up to $\mathcal{O}\left(\alpha_{s}^{2}\right)[1-3]$.

At four-loop order the two-point correlators can be considered in two limits. In the high energy limit massless propagators need to be calculated and in the low energy limit vacuum diagrams (tadpole integrals without dependence on the external momentum) arise. The evaluation of these massive tadpoles in three-loop approximation has been pioneered in ref. [4] and automated in ref. [5].

Similar to the three-loop case, the analytical evaluation of four-loop tadpole integrals is based on the traditional Integration-By-Parts (IBP) method. In contrast to the three-loop case the manual construction of algorithms to reduce arbitrary diagrams to a small set of master integrals is replaced by Laporta's algorithm $[6,7]$. In this context the IBP identities are generated with numerical values for the powers of the propagators and the irreducible scalar products. In the next step, the resulting system of linear equations is

\footnotetext{
*Talk given at the 12th International Conference on Quantum Chromodynamics, Montpellier, 4-8th July 2005

†On leave from Institute for Nuclear Research of the Russian Academy of Sciences, Moscow, 117312, Russia.
}

then solved in the next step by expressing systematically complicated integrals in terms of simpler ones. The resulting solutions are then substituted into all the other equations.

This reduction has been implemented in an automated FORM3 $[8,9]$ based program in which partially ideas described in ref. $[7,10,11]$ have been implemented. The rational functions in the space-time dimension $d$, which arise in this procedure, are simplified with the program FERMAT [12]. The automated exploitation of all symmetries of the diagrams by reshuffling the powers of the propagators of a given topology in a unique way strongly reduces the number of equations which need to be solved.

In general, the tadpole diagrams encountered during our calculation contain both massive and massless lines. In contrast, the computation of the four-loop $\beta$-functions can be reduced to the evaluation of four-loop tadpoles composed of completely massive propagators. These special cases have been considered in $[11,13,14]$.

The outline of this paper is as follows. In section 2 we discuss the calculation of the lowest expansion coefficient of the vacuum polarization function and present the results at four-loop order using methods as described above. In section 3 we present new results for the decoupling relation at four-loop order in perturbative QCD. Our conclusions are presented in section 4 


\section{Vacuum polarization function}

The vacuum polarization tensor $\Pi^{\mu \nu}(q)$ is defined as

$\Pi^{\mu \nu}(q)=i \int d x e^{i q x}\left\langle 0\left|T j^{\mu}(x) j^{\nu}(0)\right| 0\right\rangle$,

where $q^{\mu}$ is the external momentum and $j^{\mu}$ is the electromagnetic current of a heavy quark with mass $m_{h}$. The tensor $\Pi^{\mu \nu}(q)$ can be expressed by a scalar function, the vacuum polarization function $\Pi\left(q^{2}\right)$ through

$\Pi^{\mu \nu}(q)=\left(q^{\mu} q^{\nu}-q^{2} g^{\mu \nu}\right) \Pi\left(q^{2}\right)+q^{\mu} q^{\nu} \Pi_{L}\left(q^{2}\right)$.

The longitudinal part $\Pi_{L}\left(q^{2}\right)$ vanishes due to the Ward identity. The polarization function $\Pi\left(q^{2}\right)$ is related to the experimentally measurable $R$-ratio $R(s)$ through the dispersion relation:

$\Pi\left(q^{2}\right)=\Pi\left(q^{2}=0\right)+\frac{q^{2}}{12 \pi^{2}} \int d s \frac{R(s)}{s\left(s-q^{2}\right)}$.

Performing the $n$-th derivative of eq. (3) with respect to $q^{2}$ at $q^{2}=0$ one obtains the moments $\mathcal{M}_{n}^{\text {exp }}$, which can be determined experimentally:

$\mathcal{M}_{n}^{\exp }=\int d s \frac{R(s)}{s^{n+1}}=\left.\frac{12 \pi^{2}}{n !}\left(\frac{d}{d q^{2}}\right)^{n} \Pi\left(q^{2}\right)\right|_{q^{2}=0}$.

The derivatives of the polarization function on the rhs are related to the Taylor expansion coefficients $\bar{C}_{n}$ :

$\bar{\Pi}\left(q^{2}\right)=\frac{3 Q_{q}^{2}}{16 \pi^{2}} \sum_{n \geq 0} \bar{C}_{n} z^{n}$,

$\left(z=q^{2} /\left(4 \bar{m}_{h}^{2}\right)\right)$ which can be calculated in perturbative QCD. The first and higher derivatives are important for a precise determination of the charm- and bottom-quark mass (see e.g. [15]). But also the lowest expansion coefficient $\bar{C}_{0}$ has an interesting physical meaning: it relates the coupling of electromagnetic interaction in different renormalization schemes. In the case of QEDon-shell-renormalization the residue of the photon propagator is one and the electrical charge $e$ coincides with the classical limit. If one performs renormalization in the $\overline{\mathrm{MS}}$-scheme one obtains a relation between the coupling constant of the electromagnetic interaction $\alpha_{\mathrm{em}}=e^{2} /(4 \pi)$ in
QED-on-shell-renormalization and the coupling constant $\bar{\alpha}_{\mathrm{em}}=\bar{e}^{2} /(4 \pi)$ in the $\overline{\mathrm{MS}}$-scheme:

$\alpha_{\mathrm{em}}=\frac{\bar{\alpha}_{\mathrm{em}}}{1+\bar{e}^{2} \bar{\Pi}\left(q^{2}=0\right)}$.

For massive quarks, interacting through gluons, $\bar{\Pi}\left(q^{2}=0\right)$ has been evaluated in ref. [1]. At threeloop order in perturbative QCD this relation has already been determined in ref. [1]. For the QED case the corresponding result was calculated in ref. [4].

The first Taylor coefficient $\bar{C}_{0}$ has been calculated using the methods described in section 1 All tadpole diagrams were expressed through the set of 13 master integrals shown in figure 1 These master integrals have been calculated in

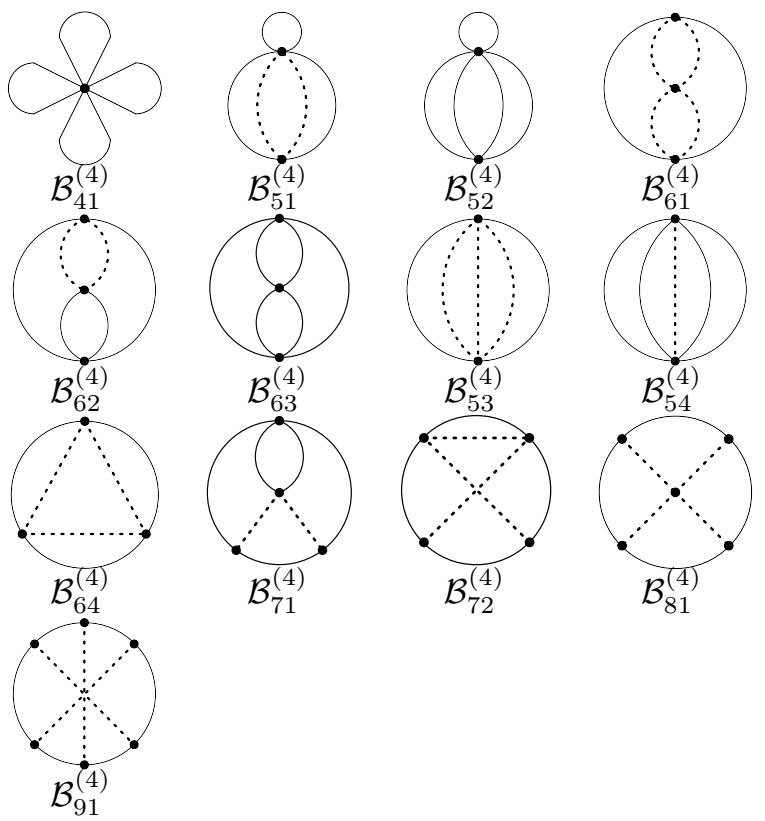

Figure 1. Master integrals. The solid lines denote massive lines, whereas the dashed lines denote massless lines.

refs. [16-18]. Inserting the master integrals into the lowest Taylor coefficient of the polarization function and performing the renormalization in 
the $\overline{\mathrm{MS}}$-scheme one obtains the following result for the expansion coefficient $\bar{C}_{0}\left(\mu=\bar{m}_{h}\right)$ :

$$
\begin{aligned}
\bar{C}_{0} & =\left(\frac{\alpha_{s}}{\pi}\right) 1.4444+\left(\frac{\alpha_{s}}{\pi}\right)^{2}\left(0.3714 n_{l}+1.725\right) \\
& +\left(\frac{\alpha_{s}}{\pi}\right)^{3}\left(0.0257 n_{l}^{2}-1.186 n_{l}-1.955\right),
\end{aligned}
$$

where $\alpha_{s}$ is the strong coupling constant in the $\overline{\mathrm{MS}}$-scheme and the symbol $n_{l}$ denotes the number of light quarks considered as massless. The part of the four-loop contribution proportional to the number $n_{f}^{2}$ of active quarks has been calculated previously up to the first physical moment $\bar{C}_{1}$ in ref. [16]. The terms proportional to $\alpha_{s}^{j} n_{l}^{j-1}$ are even known to all orders $j[19]$.

In ref. $[20,21]$ the terms being proportional to $n_{f}$ have been calculated through an independent numerical method, which is based on the Padé approximation [22-24]. These results are in complete agreement with those of eq. (6).

\section{Decoupling relation}

The masses of the known quark species differ vastly in their magnitude. Often the mass of a heavy quark $h$ is much larger than the characteristic momentum scale $\sqrt{s}$ of the reaction under consideration. In such a two interrelated problems appear when using an MS-like renormalization scheme.

First, two large but quite different mass scales, $\sqrt{s}$ and $m_{h}$, lead to two different types of potentially dangerously large logarithms of $\sqrt{s} / \mu$ and $m_{h} / \mu$ and the standard trick of a proper choice of the renormalization scale $\mu$ is no longer applicable;

Second, according to the Appelquist-Carazzone theorem [25] the effects due to heavy particles eventually should in general 'decouple' from lowenergy physics. However, a peculiarity of massindependent renormalization schemes is that the decoupling theorem does not hold in its naive form for theories renormalized in such schemes. The effective QCD action that appears will not be not canonically normalized. Large mass logarithms appear, when one calculates a physical observable.

Fortunately, both problems are controlled once the expansion parameters are properly choosen and renormalization group improvement is performed [26-29]. To be specific, consider QCD with $n_{l}=n_{f}-1$ light quarks and one heavy quark $h$ with mass $m_{h}$. The effective coupling constant $\alpha_{s}^{\prime}$ is then expressed in terms of the one of the full theory through:

$\alpha_{s}^{\prime}(\mu)=\alpha_{s}(\mu) \zeta_{g}^{2}\left(\alpha_{s}(\mu), x\right), \quad x=\log \left(\mu^{2} / \bar{m}_{h}^{2}\right),(7)$

where $\zeta_{g}$ is the decoupling function and $\bar{m}_{h}(\mu)$ is the $\overline{\mathrm{MS}}$ running mass of the heavy quark. The decoupling function is known up to three-loop order $[30,31]$. Its calculation can be reduced to the solution of vacuum integrals [30]. Thus the methods described in section 1 can be applied to calculate the decoupling function at four-loop order. The same master integrals as those shown in figure 1 appear. For a renormalization scale $\mu=\bar{m}_{h}(\mu)$ one obtains the following numerical result ${ }^{3}$ :

$$
\begin{aligned}
\zeta_{g}^{2} & =1+\left(\frac{\alpha_{s}}{\pi}\right)^{2} 0.1528 \\
& +\left(\frac{\alpha_{s}}{\pi}\right)^{3}\left(0.9721-0.0847 n_{l}\right) \\
& +\left(\frac{\alpha_{s}}{\pi}\right)^{4}\left(5.1703-1.001 n_{l}-0.0220 n_{l}^{2}\right) .
\end{aligned}
$$

The knowledge of this decoupling function is of phenomenological importance, because it allows the determination of $\alpha_{s}\left(M_{Z}\right)$ at the $Z$-Boson scale through evolution of the measured value of $\alpha_{s}\left(m_{\tau}\right)$ at the $\tau$-lepton scale. A careful analysis of the effects of four-loop running and three-loop matching on the extraction of $\alpha_{s}^{(5)}\left(M_{Z}\right)$ defined for 5 active quark flavors from $\alpha_{s}^{(3)}\left(m_{\tau}\right)$ defined for 3 active quark flavors has been recently performed [34]. We have checked that the inclusion of the newly computed four-loop matching condition leads to a further reduction of the theoretical error from the evolution.

\footnotetext{
${ }^{3}$ After the completion of our calculation we have been informed that an independent calculation of the matching function $\zeta_{g}^{2}$ at the four-loop level has been recently finished by Y. Schröder and M. Steinhauser. The same is true for $\bar{C}_{0}$ of eq. (6). Their results are in full agreement with ours. Both calculations have been made reecntly available in $[32,33]$.
} 


\section{Conclusion}

We have calculated the lowest Taylor expansion coefficient of the vacuum polarization function and the decoupling relation at four-loop order in perturbative QCD. The lowest Taylor expansion coefficient relates the electromagnetic coupling in the on-shell and in the $\overline{\mathrm{MS}}$ renormalization schemes. The decoupling relation is important for the determination of $\alpha_{s}^{(5)}\left(M_{Z}\right)$ through evolution from the measured value of $\alpha_{s}^{(3)}\left(m_{\tau}\right)$.

\section{Acknowledgments:}

We would like to thank M. Faisst for discussions about the Padé approach and M. Tentyukov for great help in dealing with FORM3 and FERMAT. K. Ch. is grateful to M. Steinhauser for useful discussions of the decoupling relations. C.S. would like to thank the Graduierten Kolleg "Hochenergiephysik und Teilchenastrophysik" for financial support. This work was supported by the Sonderforschungsbereich Transregio 9.

\section{REFERENCES}

1. K. G. Chetyrkin, J. H. Kühn and M. Steinhauser, Nucl. Phys. B482 (1996) 213.

2. K. G. Chetyrkin, R. Harlander and M. Steinhauser, Phys. Rev. D58 (1998) 014012.

3. K. G. Chetyrkin, J. H. Kühn and M. Steinhauser, Nucl. Phys. B505 (1997) 40.

4. D. J. Broadhurst, Z. Phys. C54 (1992) 599.

5. M. Steinhauser, Comput. Phys. Commun. 134 (2001) 335.

6. S. Laporta and E. Remiddi, Phys. Lett. B379 (1996) 283.

7. S. Laporta, Int. J. Mod. Phys. A15 (2000) 5087.

8. J. A. M. Vermaseren, New features of FORM, math-ph/0010025 (2000) .

9. J. A. M. Vermaseren, Nucl. Phys. Proc. Suppl. 116 (2003) 343.

10. P. Mastrolia and E. Remiddi, Nucl. Phys. Proc. Suppl. 89 (2000) 76.

11. Y. Schröder, Nucl. Phys. Proc. Suppl. 116 (2003) 402.

12. R. H. Lewis, Fermat's User Guide, http://www.bway.net/ lewis/.
13. T. van Ritbergen, J. A. M. Vermaseren and S. A. Larin, Phys. Lett. B400 (1997) 379.

14. M. Czakon, Nucl. Phys. B710 (2005) 485.

15. J. H. Kuhn and M. Steinhauser, Nucl. Phys. B619 (2001) 588.

16. K. G. Chetyrkin, J. H. Kühn, P. Mastrolia and C. Sturm, Eur. Phys. J. C40 (2005) 361.

17. Y. Schröder and A. Vuorinen, JHEP 06 (2005) 051.

18. K. Chetyrkin, M. Faisst, C. Sturm and M. Tentioukov, $\epsilon$-Finite Basis of Master Integrals for the Integration-By-Parts Method, in preparation .

19. A. G. Grozin and C. Sturm, Eur. Phys. J. C40 (2005) 157.

20. M. Faisst, Ph.D. thesis, Karlsruhe University (Cuvillier Verlag, Göttingen, 2005) .

21. M. Faisst, K. G. Chetyrkin and J. H. Kühn, Nucl. Phys. Proc. Suppl. 135 (2004) 307.

22. J. Fleischer and O. V. Tarasov, Z. Phys. C64 (1994) 413.

23. J. Fleischer and O. V. Tarasov, Nucl. Phys. Proc. Suppl. 37B (1994) 115.

24. D. J. Broadhurst, P. A. Baikov, V. A. Ilyin, J. Fleischer, O. V. Tarasov and V. A. Smirnov, Phys. Lett. B329 (1994) 103.

25. T. Appelquist and J. Carazzone, Phys. Rev. D11 (1975) 2856.

26. S. Weinberg, Phys. Lett. B91 (1980) 51.

27. W. Wetzel, Nucl. Phys. B196 (1982) 259.

28. W. Bernreuther and W. Wetzel, Nucl. Phys. B197 (1982) 228.

29. W. Bernreuther, Ann. Phys. 151 (1983) 127.

30. K. G. Chetyrkin, B. A. Kniehl and M. Steinhauser, Nucl. Phys. B510 (1998) 61.

31. K. G. Chetyrkin, B. A. Kniehl and M. Steinhauser, Phys. Rev. Lett. 79 (1997) 2184.

32. K. G. Chetyrkin, J. H. Kuhn and C. Sturm, hep-ph/0512060.

33. Y. Schroder and M. Steinhauser, JHEP 01 (2006) 051.

34. M. Davier, A. Hocker and Z. Zhang, hep-ph/0507078. 